OPEN ACCESS

Edited by:

Elvira Larqué

Universidad de Murcia, Spain

Reviewed by:

Maria Caterina Mione

University of Trento, Italy

Alice Hudder

Lake Erie College of Osteopathic

Medicine, United States

Maria Luisa Cayuela,

Hospital Universitario Virgen de la

Arrixaca, Spain

*Correspondence:

Letizia Pitto

I.pitto@ifc.cnr.it

${ }^{\dagger}$ Present Address:

Elena Chiavacci,

International Centre for Genetic

Engineering and Biotechnology,

Trieste, Italy

Specialty section:

This article was submitted to

Epigenomics and Epigenetics,

a section of the journal

Frontiers in Cell and Developmental

Biology

Received: 21 November 2017

Accepted: 15 May 2018

Published: 05 June 2018

Citation:

Guzzolino E, Chiavacci E, Ahuja N,

Mariani L, Evangelista M, Ippolito $C$,

Rizzo M, Garrity D, Cremisi F and

Pitto L (2018) Post-transcriptional

Modulation of

Sphingosine-1-Phosphate Receptor 1

by miR-19a Affects Cardiovascular

Development in Zebrafish.

Front. Cell Dev. Biol. 6:58

doi: 10.3389/fcell.2018.00058

\section{Post-transcriptional Modulation of Sphingosine-1-Phosphate Receptor 1 by miR-19a Affects Cardiovascular Development in Zebrafish}

\author{
Elena Guzzolino ${ }^{1,2}$, Elena Chiavacci ${ }^{1+}$, Neha Ahuja ${ }^{3}$, Laura Mariani ${ }^{1}$, Monica Evangelista ${ }^{1}$, \\ Chiara Ippolito ${ }^{4}$, Milena Rizzo ${ }^{5}$, Deborah Garrity ${ }^{3}$, Federico Cremisi ${ }^{6}$ and Letizia Pitto ${ }^{1 *}$ \\ ${ }^{1}$ Institute of Clinical Physiology, National Research Council, Pisa, Italy, ${ }^{2}$ Institute of Life Sciences, Scuola Superiore \\ Sant'Anna, Pisa, Italy, ${ }^{3}$ Department of Biology, Center for Cardiovascular Research, Colorado State University, Fort Collins, \\ CO, United States, ${ }^{4}$ Department of Clinical and Experimental Medicine, University of Pisa, Pisa, Italy, ${ }^{5}$ Tumour Institute of \\ Tuscany, Florence, Italy, ${ }^{6}$ Scuola Normale Superiore di Pisa, Pisa, Italy
}

Sphingosine-1-phosphate is a bioactive lipid and a signaling molecule integrated into many physiological systems such as differentiation, proliferation and migration. In mammals S1P acts through binding to a family of five trans-membrane, G-protein coupled receptors (S1PRs) whose complex role has not been completely elucidated. In this study we use zebrafish, in which seven s1prs have been identified, to investigate the role of s1pr1. In mammals S1PR1 is the most highly expressed S1P receptor in the developing heart and regulates vascular development, but in zebrafish the data concerning its role are contradictory. Here we show that overexpression of zebrafish s1pr1 affects both vascular and cardiac development. Moreover we demonstrate that s1pr1 expression is strongly repressed by miR-19a during the early phases of zebrafish development. In line with this observation and with a recent study showing that miR-19a is downregulated in a zebrafish Holt-Oram model, we now demonstrate that s1pr1 is upregulated in heartstring hearts. Next we investigated whether defects induced by s1pr1 upregulation might contribute to the morphological alterations caused by Tbx5 depletion. We show that downregulation of s1pr1 is able to partially rescue cardiac and fin defects induced by Tbx 5 depletion. Taken together, these data support a role for s 1 pr 1 in zebrafish cardiovascular development, suggest the involvement of this receptor in the Tbx5 regulatory circuitry, and further support the crucial role of microRNAs in early phase of zebrafish development.

Keywords: sphingosine-1-phosphate receptor 1, microRNA, zebrafish, cardiovascular development, Holt Oram syndrome

\section{INTRODUCTION}

Sphingosine-1-phosphate (S1P) is a biologically active lysophospholipid with crucial role for membrane structure and function in eukaryotes. In vertebrates, S1P is found in the extracellular environment and interacts with cell-surface receptors to regulate an array of cellular responses, including cellular differentiation, proliferation, migration, cytoskeletal reorganization and apoptosis (Chun et al., 2002; Blaho and Hla, 2011). In particular, S1P action is implicated 
in the regulation of numerous cardiovascular processes including angiogenesis, vascular permeability, arteriogenesis, cardiac function, vascular development, and vascular tone (Levade et al., 2001; Allende and Proia, 2002; Alewijnse et al., 2004). Moreover, the involvement of S1P in mouse limb development (Chae et al., 2004) and neurogenesis (Mizugishi et al., 2005) has been also reported. After release from cells in response to various cellular stimuli, S1P acts in an autocrine and paracrine manner through its cell surface receptors. S1P receptors (S1PRs) are G protein-coupled receptors (GPCRs) critical for S1P action. In mammals, five S1prs (S1pr1-S1pr5) have been identified. Three S1P receptor subtypes $(S 1 p r 1,2,3)$ are expressed in the adult cardiovascular system each with a unique pattern of expression (Alewijnse et al., 2004; Means and Brown, 2009).

In mammals, S1pr1 is the most highly expressed S1P receptor in the cardiac myocytes in the developing heart; it also regulates vascular development in coordination with S1pr2 and S1pr3. S1pr1 knockout (KO) mice show intrauterine lethality between E12.5 and E14.5 because of severe hemorrhage (Liu et al., 2000). Recently analysis of S1pr1 LacZ knockin embryos revealed that S1P signaling via S1P1 in cardiomyocytes plays a previously unknown and necessary role in heart development in mice (Clay et al., 2016).

In zebrafish the s1pr2 (miles apart) gene has been shown to play a crucial role in heart development by affecting the migration of myocardial precursor cells to the ventral midline of the embryo where they assemble into the heart tube. In accord with this function, deletion of s1pr2 results in cardia bifida (Kupperman et al., 2000). In contrast, data concerning the s1prl function in zebrafish are rather controversial: several papers in recent years highlighted the s1pr1 role in vascular development and in controlling the venous endothelial barrier integrity, similar to the role played by this receptor in mouse (Ben Shoham et al., 2012; Gaengel et al., 2012; Tobia et al., 2012; Mendelson et al., 2013). All these data were obtained by morpholinomediated loss of function experiments. However a recent paper by Hisano et al. analyzed all of the available slpr zebrafish mutants generated by TALEN-mediated frameshift mutations. They demonstrated that none of the slpr mutants showed developmental defects with the exception of s1pr2 mutant which exhibits embryonic lethality arising from its cardiac defect (Hisano et al., 2015). These data suggest a previously unrevealed redundancy in functions of the $\mathrm{S} 1 \mathrm{P}$ receptor-mediated signaling in zebrafish similarly to the partially overlapping expression of $\mathrm{S} 1 \mathrm{P}$ receptors observed in mouse (Means and Brown, 2009).

\footnotetext{
Abbreviations: tbx5, T-box transcription factor 5; hst, hearstring; HOS, HoltOram syndrome; S1P, shingosine-1-phosphate; s1pr1, sphingosine-1-phosphate receptor 1; GPCRs, G protein-coupled receptors; wt, wild type; CDS, coding sequence; UTR, untranslated region; Scr, scramble; MO, morfolino; ef1 $\alpha$, elongation factor $1 \alpha$; nppa, natriuretic peptide $\mathrm{A}$; hand2, heart and neural crest derivates expressed 2; mef2ca, myocyte enhancer factor 2ca; mef2aa, myocyte enhancer factor 2aa; cx43, connection 43; cyp26b1, cytochrome P450, family 26, subfamily b, 1; gata4, GATA binding protein 4; cmlc2, cardiac myosin light chain 2; vmhc, ventricular myosin heavy chain; ISV, intersegmental vessel; CVP, caudal vein plexus; hpf, hours post fertilization; RFP, red fluorescent protein; GFP, green fluorescent protein; ddPCR, digital droplet PCR; RT-PCR, Real time PCR.
}

In this study, to further investigate the role of s1pr1 in zebrafish and to work around the redundancy problem, we overexpressed this receptor during early developmental stages. Our data support the involvement of s1pr1 in cardiovascular development. Importantly, we show that s1prl during the early zebrafish developmental stages is controlled by miR-19a and as such might be part of the Tbx $5 / \mathrm{miR}-19$ a regulatory circuit affecting heart development.

\section{MATERIALS AND METHODS}

\section{Reagents}

Mature dre-miR19a-3p mimic (F 5'-UGUGCAAAUCUAUGC AAAACUGAUU- $3^{\prime}$ and $\mathrm{R} 5^{\prime}$-UCAGUUUUGCAUAGAUUU GCUAAUU- $3^{\prime}$ ) and a miR-Ct (F $5^{\prime}$-CUCUAGGUUAAACUC CUGGUU- $3^{\prime}$ and $\mathrm{R} 5^{\prime}$-AACCAGGAGUUUAACCUAAUGUU$\left.3^{\prime}\right)$ were synthesized by GenePharma (Shanghai, China). Tbx5a morpholino(5'-GAAAGGTGTCTTCACTGTCCGCCAT-3', Chiavacci et al., 2012) and s1pr1 morpholino (5'-AGTGTC TGGCGATTAGGTCATCCAT-3', Mendelson et al., 2013) were synthesized by Gene Tools (LLC USA.). QuikChange II XL sitedirected mutagenesis kit (Agilent), $\alpha$-minimal Essential Medium (Invitrogen, Life Technologies Italia, Monza, Italy); Polyfect, miRNeasy Mini Kit, miScript Reverse Transcription kit and Quantitec Reverse Transcription kit, (QIAGEN, Milan, Italy); pGEMTeasy vector andDual-Luciferase Reporter Assay System (PROMEGA); $\mathrm{pCS}^{+}$vector (Addgene); zebrafish diet (SDS, Dietex,France); mMESSAGEmMACHINE SP6 transcription Kit (Thermo Fisher); (R)-3-amino-4-(3-hexylphenylamino)-4oxobutylphosphonic acid trifluoroacetate (W146) (Avanti, Polar Lipid. Inc. USA).

\section{Zebrafish Lines}

Wild-type AB, $\operatorname{Tg}(f l k 1: E G F P), \operatorname{Tg}(M y l 7: E G F P)$ and the $T b x 5 a^{\text {s296 }}$ mutant lines were used in these studies. Zebrafish were raised and maintained under standard laboratory conditions (Westerfileld M zebrafish book) in Zebrafish Housing Systems (Tecniplast, Varese, Italy). The local ethics committee approved animal studies and all procedures conformed to the essential ethical rules and the current applicable legislation. Adult zebrafish were bred under standard conditions and embryos obtained by natural spawning and incubated at $28.5^{\circ} \mathrm{C}$ in E3 medium (Westerfield M. zebrafish book). They were further staged and fixed at specific time-points as described by Kimmel (Kimmel et al., 1995). When animals need to be euthanized, an overdose of tricaine methane sulfonate (200-300 $\mathrm{mg} \mathrm{l}^{-1}$ ) by prolonged immersion was used, which is a well-established humane method.

\section{Zebrafish Microinjection}

Transgenic $T g(M y l 7: e G F P)$ or $T g(f l k 1: E G F P)$ zebrafish embryos were injected at the 1 cell stage with a constant injection volume ( $\sim 1 \mathrm{nl}$, confirmed by volume analysis) using a microinjector made by Tritech Research (Los Angeles USA).

\section{Cells Culture and Transfection}

HEK-293 cells were grown in DMEM $+10 \%$ FBS, $2 \mu \mathrm{g} / \mathrm{ml}$ L-glutamine and $50 \mu \mathrm{g} / \mathrm{ml}$ streptomycin at $37^{\circ} \mathrm{C}$ in a humidified 
atmosphere containing $6 \% \mathrm{CO}_{2}$. Cells were seeded at a density of $1.5 \times 10^{5}$ cells per well in 12 well dishes and grown for $24 \mathrm{~h}$. Twenty-four hours later, cells were transfected using Polyfect (Qiagen) as transfectant, according to the manufacturer's recommendations. In each transfection $100 \mathrm{ng} / \mu \mathrm{l}$ of the s1pr1$3^{\prime}$ UTR-wt construct, the s1pr1-3'UTR-mut, containing the mutation in the miR-19a binding site, or the pGLU empty vector as control were co-trasfected with increasing doses of duplex simiRNA19a in the presence of $100 \mathrm{ng} / \mu \mathrm{l}$ of Renilla expressing plasmid as internal standard. In each transfection the total amount of the transfected si-miRNA was kept constant by adding a scrambled miRNA (miR-Ct) to the specific miR-19a to obtain $80 \mathrm{ng}$ si-miRNA concentration. After $24 \mathrm{~h}$ at $37^{\circ} \mathrm{C}$ cells were washed with PBS for two times and processed for the Luciferase assay (Verduci et al., 2010).

\section{s1pr1 Cloning and In Vitro Transcription}

The s1pr 1 CDS and full-length clones were obtained using cDNA generated from total RNA of $48 \mathrm{hpf}$ wild type zebrafish embryos. The s1pr 1 CDS was PCR amplified using the following primers: F: 5'-ATGGATGACCTAATCGC- ${ }^{\prime}$ and R: 5'-ACGACAAAGTTC ACGAATAGTC- $3^{\prime}$. To generate the full-length clone including the 854 bp of the 3 'UTR, a different reverse primer was used Rfull: $5^{\prime}$-GAACAGGGACAAAACTGGCTC- $3^{\prime}$. Following ligation into the pGEM T-easy vector, the inserts were subcloned into the PCS2 vector and verified by sequencing. The inserts were linearized with NotI and capped mRNAs were generated using the mMESSAGEmMACHINE SP6 transcription Kit. The same kit was used to in vitro transcribe the RFP CDS from the NotI linearized $\mathrm{pCS} 2+$ vector.

The s1pr1 $3^{\prime} \mathrm{UTR}$ was PCR amplified using the following primers: F: 5'-GGTACCTCTTCTTCTTAAAGC-3' (where italics indicates the bases added to generate the restriction site KpnI) and R: 5'-GAACAGGGACAAAACTGGCTC- $3^{\prime}$. Following ligation into the pGEM T-easy vector, the insert was excised using KpnI/SalI, subcloned into KpnI/XhoI of the pGLU Dual-luciferase reporter plasmid (Poliseno et al., 2010) and verified by sequencing. The mutated version of the s1pr1 $3^{\prime} \mathrm{UTR}$ (s1pr1-3'UTR-mut) was generated utilizing the s1pr1-3'UTR $w t$ plasmid as template and modifying the miR-19a seed binding site using the QuikChange II XL site-directed mutagenesis kit. To generate the GFP sensor reporter vector for zebrafish injection the s1pr1 3'UTR was excised from pGLU-s1pr1-3'UTR vector using $\mathrm{XbaI}$ and the insert was subcloned into XbaI linearized pCS2-GFP vector. The correct insert orientation was checked by restriction digestion analysis.

\section{s1pr1 Chemical Inhibition in Zebrafish Embryos}

W146 was dissolved in a solution of 20\% 2-hydroxypropylbeta-cyclodextrin (Tarrason et al., 2011) and $50 \mathrm{mM} \mathrm{NaCO} 2$ at the stock concentration of $100 \mu \mathrm{M} / \mathrm{ml}$. $24 \mathrm{hpf}$ non-injected and Tbx5 morphant embryos were exposed to W146 at the following concentrations: $0,0.00025,0.005,0.05,0.5$, and $1 \mu \mathrm{g} / \mathrm{ml}$. We started from $0.00025 \mu \mathrm{g} / \mathrm{ml}$ since the $0.005 \mu \mathrm{g} / \mathrm{ml}$ is the concentration sufficient to cause vessel defects when injected intravenously in mouse (Tarrason et al., 2011) but we were not able to use a dose higher than $1 \mu \mathrm{g} / \mathrm{ml}$ due to solubilization problem. The quantity of solvent added to E3 medium was kept constant among the different treatments. Embryos were scored at $72 \mathrm{hpf}$ for heart phenotypes, fin phenotypes, as well as heart rate. For heart rate, each fish was scored three times. Heart beats were counted for either 10 or $20 \mathrm{~s}$, and average heartbeats/min was calculated. Statistics were run on the average heartbeats/min of the three trials.

\section{Dual-Luciferase Reporter Assay and In Vivo GFP Assay}

Luciferase activity was measured $24 \mathrm{~h}$ after transfection using the Dual-Luciferase Reporter Assay system. HEK 293 cells after PBS washing were extracted by addiction of $100 \mu$ l Passive Lysis Buffer 1x (Promega) in each dish. After $5 \mathrm{~min}$ at room temperature the plate was put at $-80^{\circ} \mathrm{C}$ for $10 \mathrm{~min}$. The contents of each dish was collected and centrifuged for $10 \mathrm{~min}$ at $4^{\circ} \mathrm{C}$. The obtained supernatant was immediately assayed or stored at $-80^{\circ} \mathrm{C}$. Firefly luciferase activity was normalized to Renilla activity for each transfected dish. Assays were performed in three independent experiments.

For the in vivo sensor assay, approximately $500 \mathrm{pg}$ of the pCS2-GFP mRNA was coinjected with either si-miR-19a or control mimic $(10 \mu \mathrm{M})$ into single-cell stage (1 $\mathrm{nl}$ injection volume) embryos. $24 \mathrm{hpf}$ after microinjection embryos were analyzed by fluorescent microscopy.

\section{Imaging}

Staining was observed with Leica M80 microscope and images were acquired with Nikon DS-Fil camera and NIS-Elements F 3.0 software. For fluorescence microscopy Leica DM IL microscope and Nikon YFL microscope both equipped with CoolSnap CF camera (Photometric) were used. Images were processed with Gimp-2.6 or ImageJ software.

Confocal imaging was performed with Leica TCS SP8 confocal laser scanning microscope (Leica Microsystems, Mannheim, Germany) equipped with Leica Application Suite (LAS) X software. The Z-stack function scanned the organisms along the " $z$ " dimension. All confocal frames were taken with a good level of resolution along with a low scanning speed and a specific setting to visualize the green signal (format $1,024 \times 1,024 \mathrm{dpi}$ resolution).

\section{Heart Dissection}

Zebrafish hearts were dissected with physical pressure using a glass pipette according to Singleman and Holtzman (2011). Embryos were previously anesthetized with tricaine $(0.04 \%)$ and physical heart dissection was performed in L15 medium with the addition of $10 \%$ sheep/bovine serum. After the dissection about 25 hearts for each lines were extracted. Three extractions from 3 different clutches were performed.

\section{RNA Extraction and Quantitative Real Time RT-PCR and Digital Droplet PCR}

Total RNA was extracted using the miRNeasy Mini kit (Qiagen). RNA was quantified using a NanoDrop-1000 spectrophotometer and quality was monitored by the Agilent 2100 Bioanalyser 
(Agilent Technologies, Santa Clara, CA). cDNA was reverse transcribed using miScript Reverse Transcription kit (for miRNA analysis) and Quantitec Reverse Transcription kit (for gene analysis). Real-time PCR (qRT-PCR) was carried out using QuantiFast SYBR Green kit with Rotor gene (Qiagen). Relative quantification was performed as previously described (Chiavacci et al., 2015). Elongation factor $\alpha(e f 1 \alpha)$ was used as the reference gene. For Digital Droplet PCR the QX200 droplet digital PCR system (BioRad) was used following the manufacturer's instruction. After PCR, read-out of positive vs. negative droplets was performed with the droplet reader and the absolute quantification of PCR target was analyzed using QuantaSoft software (BioRad). Absolute readouts were normalized to the amount of ef1a present in each sample. The list of primers used is reported in Table 1.

\section{Whole Mount in Situ Hybridization}

Whole mount In Situ Hybridization (ISH) was performed as previously described (Chiavacci et al., 2012).

\section{Statistical Analysis}

Data were analyzed using GraphPad Prism (GraphPad Software, San Diego, CA USA). Statistical differences were determined by unpaired $t$-test, and Fisher's test with values of $P<0.05$ were considered statistically significant. Each experimental point in the graph represents the mean $\pm \mathrm{SE}$ of at least three independent experiments.

\section{RESULTS}

\section{s1pr1 Overexpression Affects Heart, Fin and Vascular Development in a Dose-Dependant Way}

The coding sequence (CDS) of s1pr1 was cloned and in vitro transcribed. 150 and 300 pg of the obtained s1pr1 CDS RNA were injected in $\mathrm{Tg}(k d r l: E G F P)^{s 843}$ transgenic zebrafish embryos in which EGFP expression is driven by the promoter of the pan-endothelial marker vegfr-2 (Beis et al., 2005). As control, the RNA of Red Fluorescent Protein (RFP) obtained from in vitro transcription of the pCS2+ vector was used. As already reported, s1pr1 overexpressing (S1up) embryos show alteration in the intersegmental vessel (ISV) formation and in the caudal vein plexus (CVP) (Mendelson et al., 2013). In S1up embryos, the ISV are shorter and truncated compared to ISVs in the control (Ct) embryos and the area of CVP is more compact compared to the honeycomb-like morphology which characterizes the Ct embryos. We included in the mild phenotypes embryos showing only shorter ISV and almost normal CVP while we considered as severe embryos with truncated ISV and more compact CVP (Figure 1B). Both ISV and CVP defects show s1prl dose-dependent increase in severity (Figures 1A,B).

In order to investigate whether s1pr1 overexpression also affects cardiac development, s1pr1 CDS mRNA was injected in $\mathrm{Tg}(m y l 7: E G F P)$ embryos in which cardiomyocytes are marked with green fluorescence (Huang et al., 2003). Increasing doses of s1pr1 raised the frequency and severity of cardiac defects (Figures 2A,B). In the less severe phenotypes, only cardiac looping was affected while in the most severe phenotypes also the chamber shape showed alteration and in particular the ventricle was smaller and irregular and the atrium was dilated (Figure 2B). In a few cases, cardia bifida was evident as two small deformed beating hearts located on either side of the midline (Figure 2C). Interestingly, the occurrence of defective or absent pectoral fins paralleled the s1pr1 dose-dependent increase of cardiac defects (Figures 2D,E). Embryos injected with the same dosage of RFP mRNA showed no apparent phenotype (Figure 2B). On the contrary, downregulation of s1pr1 by morpholino injection, although strongly affecting vascular morphology as largely demonstrated in several papers (Ben Shoham et al.,

TABLE 1 | List of oligonucleotides used in this study.

\begin{tabular}{|c|c|c|}
\hline Gene & Forward primer & Reverse primer \\
\hline ef1 $\alpha$ & CTGGAGGCCAGCTCAAACAT & ATCAAGAAGAGTAGTACCGCTAGCATTAC \\
\hline nрра & CAACATGGTCAAGCTCAA & GGCTCTCTCTGATGCCTCTTC \\
\hline hand2 & AAGGCGAAAGAAGGAAATGAA & GCCAACCAGTTCTCCCTTTA \\
\hline$n k x 2.5$ & TGACACATTTGAAGACAAAGAGAAA & TССТССТСТTССTСTGСTTG \\
\hline mef2caA & GAAACACAGGAGGTCTGATGG & GTGGTTTCCGTACCCGTT \\
\hline mef2aa & GGGGACCACGGAGAAAAA & TGGCTTCAATGCCTTCTCT \\
\hline$c \times 43$ & TCGCGTACTTGGATTTGGTGA & CCTTGTCAAGAAGCCTTCCCA \\
\hline bmpr1aa & GCGTCAGCTITGTTCATCA & TGATCAGGATTCTGACCTGCT \\
\hline cyp26b1 & GCCAACTCAATAGGAGACATCC & CCAGAGCCTCATGGCTAAAAA \\
\hline gata4 & TCGCACTTCGACAGCTCCGTA & GACATCGCCCCGCAGTTCACA \\
\hline s1pr1 & TGTCAGACCCTCACCTGCT & TTCATGGCAGAGTTGAGCAC \\
\hline s1pr2 & CACGCGCTTCTTCTCTCC & CAGCCCGAAGTCACGTCT \\
\hline s1pr3a & CATACCGCAGAGAACAGCAAC & CTGACTTGGCTGCACCACTA \\
\hline s1pr4 & AACCGAAGAACGGCAAAAA & CGCTTGACGCAGATAAACAA \\
\hline s1pr5a & CATGCCGTITCTGGATTGTA & AGGCCTTCCAGCCTGTGT \\
\hline s1pr5b & AGAACCTGACGGTCCTGCT & GGTCCGATAGTGCCAGGTT \\
\hline
\end{tabular}



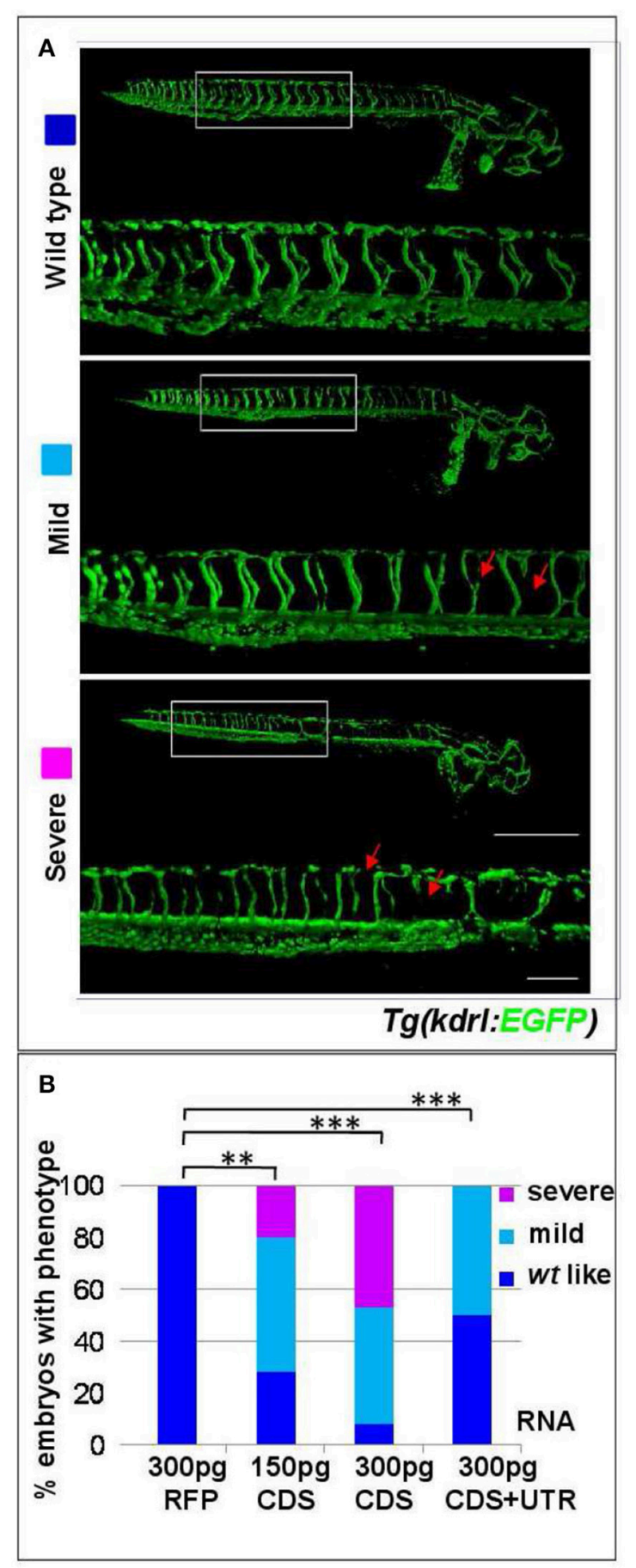

FIGURE 1 | s1pr1 overexpression affects vascular development. (A) Confocal microscopy of representative 48 hpf $T g(k d r l: E G F P)^{5843}$ embryos showing vascular defects of different severity. The $T g(k d r l: E G F P)^{5843}$ embryos were injected with in vitro transcribed and capped mRNA of the full-length $(\mathrm{CDS}+\mathrm{UTR})$ or the CDS sequence of s1pr1 at the reported doses (pg). The total numbers of embryos analyzed were as follows: 300 pg RFP CDS injected $n=20 ; 150$ pg s1pr1 CDS injected $n=22 ; 300$ pg s1pr1 CDS injected $n=38 ; 300$ pg s1pr1 CDS+UTR injected $n=15$. Red arrows indicate examples of shorter and incomplete ISVs. Scale bar $=500 \mu \mathrm{m}$ (zoom $100 \mu \mathrm{m})$. (B) Blinded quantification of the abnormal vascular phenotypes showed in (A). ${ }^{\star \star} P<0.001,{ }^{\star \star \star} P<0.0001$.
2012; Gaengel et al., 2012; Tobia et al., 2012; Mendelson et al., 2013), has a limited effect on heart development. The most frequent cardiac alteration we observed was an enlargement of atrial size, which occurred in about $20 \%$ of injected embryos (Figure S1).

To investigate the molecular consequences of increased cardiac expression of s1prl, hearts were dissected from $48 \mathrm{hpf}$ embryos previously injected with CDS mRNA of $s 1 p r 1$ or RFP as control. Total RNA was extracted from pools of 20-30 hearts in three different experiments and first of all we verified whether upregulation of s1pr1 might impact the cardiac level of the other members of the s1p receptor family. We analyzed by Q-RT PCR the expression of s1pr2, s1pr3a, s1pr3b, s1pr4, s1pr5a, and s1pr5b (Data Sheet 1) but only s1pr2 and s1pr5a showed an expression level comparable to s1pr1 in the RFP injected hearts. All the other receptors were not detectable at the cardiac level both in RFP and in s1prl injected hearts. As a consequence of s1prl overexpression we observed a significant but small upregulation of $s 1 p r 2$ while the level of $s 1 p r 5 a$ was not changed (Figure S2).

Next we quantified by Q-RT PCR several cardiac markers which characterize cellular determination, patterning and differentiation of cardiac phases (Data Sheet 1). We observed a small but significant downregulation of some important cardiac regulators such as $t b x 5$, hand2, mef2AA, and gata4 (Figure 3A). Following gastrulation, zebrafish, gata4, hand2, and tbx5 are expressed bilaterally in portions of the Lateral Plate Mesoderm (Serbedzija et al., 1998; Reiter et al., 1999; Begemann and Ingham, 2000; Ruvinsky et al., 2000) and contribute to the especially intricate process of patterning of the LPM cardiogenic region.

Both $t b x 5$ and hand 2 are involved not only in zebrafish heart but are also expressed in the fin field and contribute to pectoral fin development (Yelon et al., 2000; Garrity et al., 2002; Parrie et al., 2013). Interestingly Hand2, Mef2AA, and Gata4 proteins are all Tbx5 interactors (Ghosh et al., 2009; Lu et al., 2016). Since the alteration of heart morphology might be a consequence of the vessel organization disruption (Auman et al., 2007; Dietrich et al., 2014), by in situ hybridization we investigated whether s1pr1 overexpression might influence the myocardial precursor pool before heart tube formation. Figures 3B,C highlight a reduction of myocardial precursors, specifically ventricular primordium in the presence of an excess of s1prl supporting a role of this receptor in the cardiac context.

These data support the hypothesis that s1prl dysregulation in zebrafish heart might affect early stages of heart development.

\section{s1pr1 Is a Direct Target of miR-19a}

During the last decade, several studies by our group and others clearly showed that microRNAs are critical components of the cardiogenic regulatory network, and play numerous roles in the growth, differentiation, and morphogenesis of the developing heart (van Rooij et al., 2008; Cordes and Srivastava, 2009; Chiavacci et al., 2012; Agostini et al., 2015; D'Aurizio et al., 2016). MicroRNAs exert their activity by preferentially binding to specific sites within the 3'-UTR of the mRNA target (Bartel, 2009).

Therefore we decided to more fully investigate the impact of slprl overexpression during the early stages 

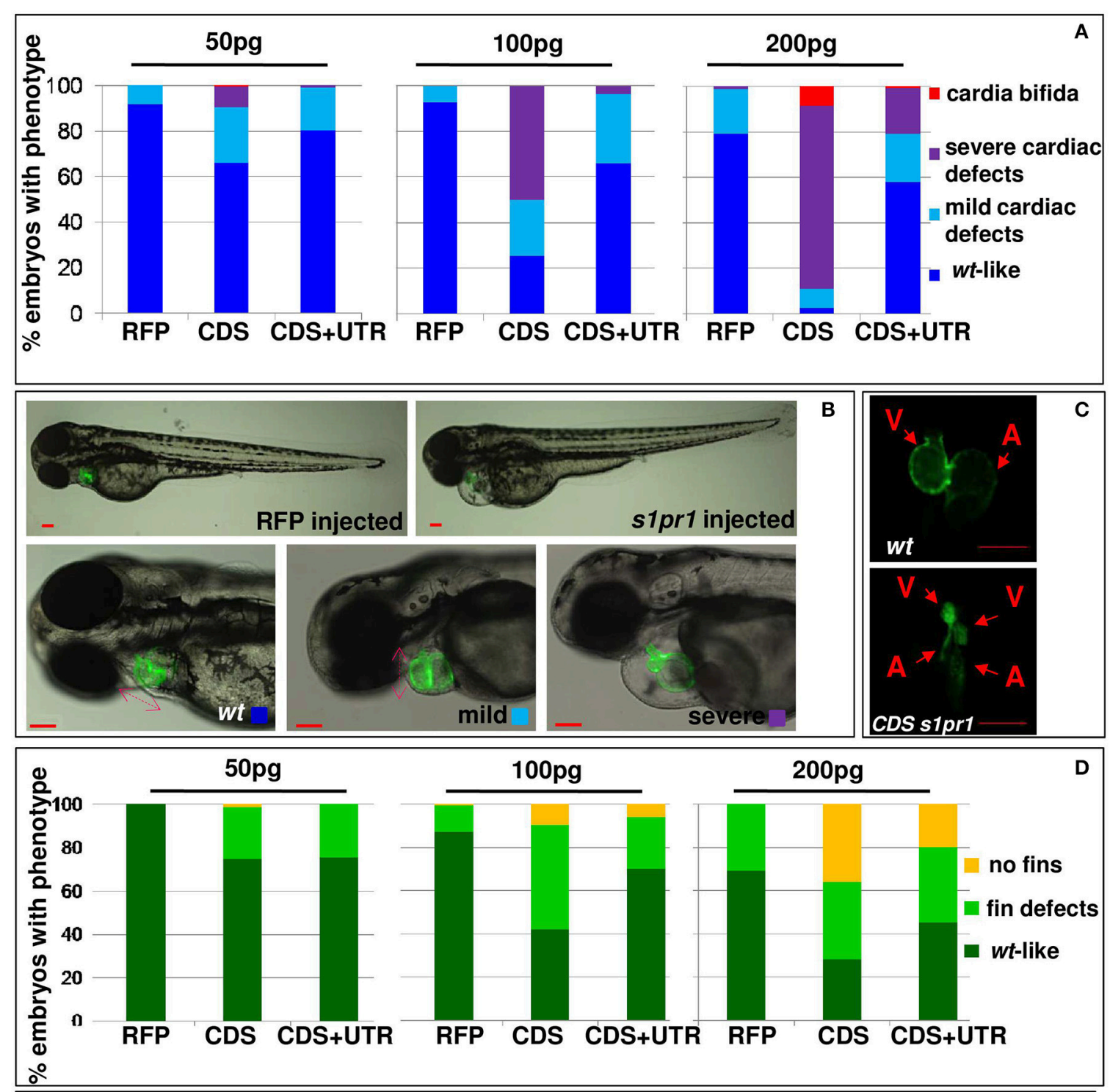

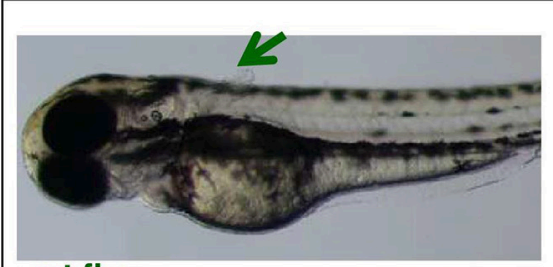

wt fins

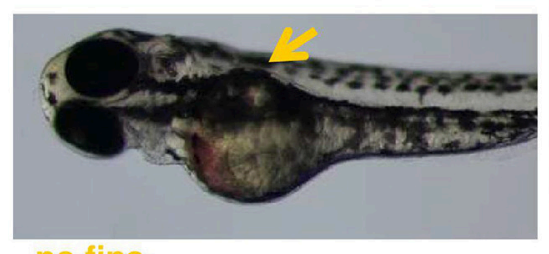

no fins

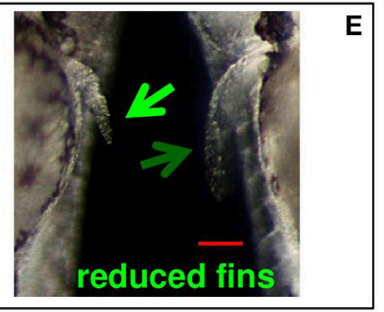

FIGURE 2 | s1pr1 overexpression affects heart and fin development in a dose-dependent way. Analysis of 72 hpf Tg(Myl7:EGFP) embryos injected with CDS or CDS+UTR mRNA of s1pr1at the reported doses (pg). The percentage of embryos with the indicated heart (A) or pectoral fin (D) defects was averaged across multiple independent experiments carried out in double blind. The total number of analyzed embryos were as follows: RFP mRNA injected $n=185$ (50 pg), $n=138$ (100 pg), $n=89$ (200 pg); s1pr1CDS mRNA injected $n=195$ (50 pg), $n=199$ (100 pg), $n=106$ (200 pg); s1pr1 CDS+UTR mRNA injected $n=214$ (50 pg), $n=198$ (100 $\mathrm{pg}), n=96$ (200 pg). For all tested doses, differences between control and s1pr1 injected embryos were significant (Fisher's test $P<0.0001)$ with the only exception of the 50 pg mRNA CDS+UTR which was not significant. (B,C,E) Images representative of the different phenotypes. In (B), the red dashed arrows emphasize the valve orientation as indicator of correct looping. In (C) an example of cardia bifida phenotype is shown. In (E) the yellow arrow indicates the absence of fins, the green arrows indicate WT fins and the pale green arrow points to an example of defective fin. V, ventricle; $a$, atrium. Red scale bar $=100 \mu \mathrm{m}$. 


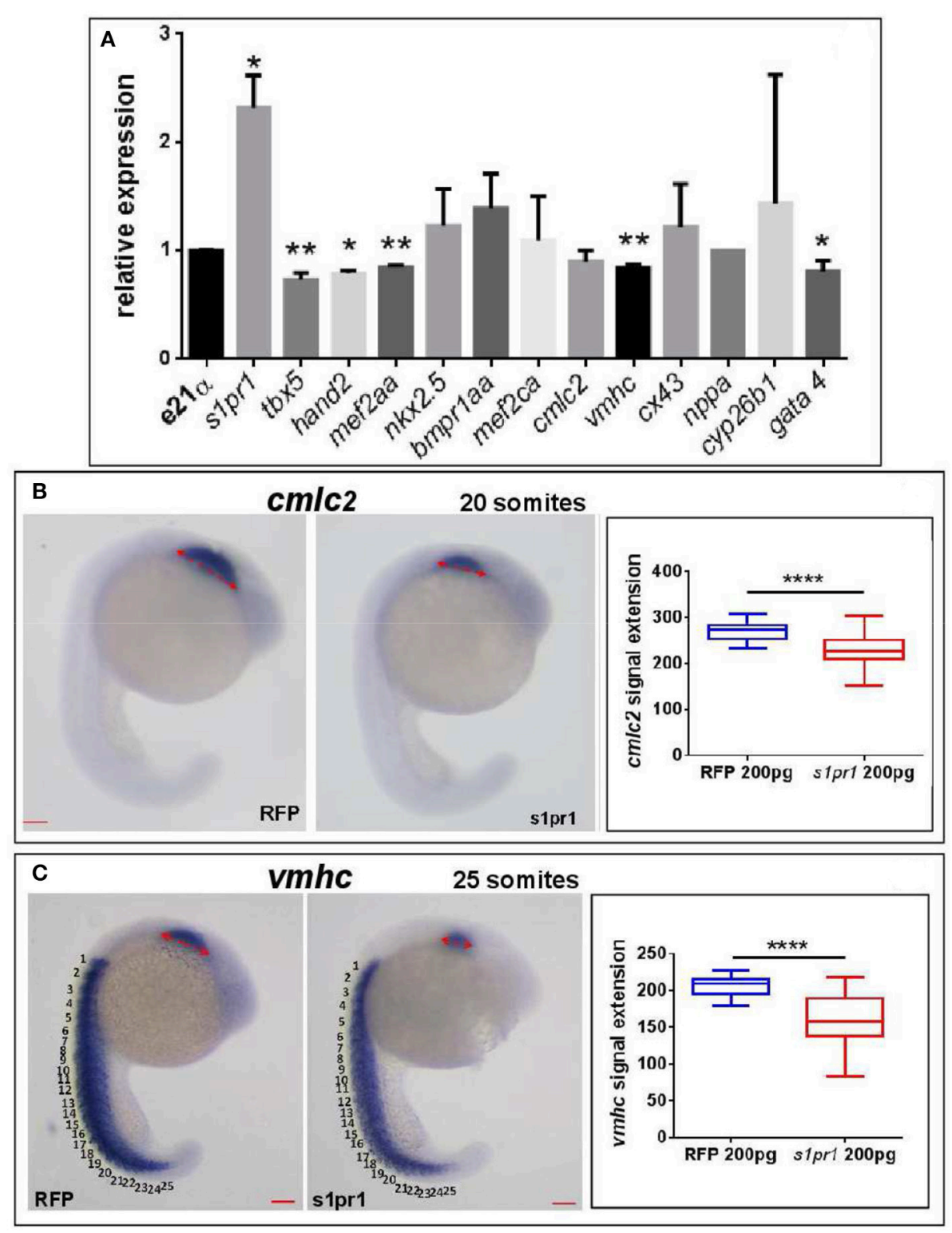

FIGURE 3 | s1pr1 overexpression significantly affects the expression of cardiac precursors and some cardiac regulators. One cell stage embryos were microinjected with $200 \mathrm{ng}$ of the CDS mRNA of s1pr1 or RFP. (A) $48 \mathrm{~h}$ after microinjection 20-30 hearts for each experiment were dissected as described in Materials and Methods section. Total RNA was extracted, reverse-transcribed and Q-RT PCR performed. Data were normalized using ef1 $\alpha$ as internal standard and are relative to values of hearts from RFP injected embryos. Three different experiments starting from 3 different clutches were used for this analysis. ${ }^{\star} P<0.05,{ }^{\star \star} P<0.001$. (B,C) $\mathrm{cmlc2}$ (B) and vmhc (C) ISH were performed on 20 (A) and 25 (B) somite stage embryos. Left side, examples of ISH: double head arrows indicate the extension of the hybridization signals. Scale bar $=100 \mu \mathrm{m}$. Right side shows the quantifications of the hybridization signals: the arrow lengths in $\mu \mathrm{m}$ are reported. About 40 embryos were analyzed for each experiment. ${ }^{* * * *} P<0.0001$.

of zebrafish development by overexpressing the full sequence of the s1pr1 transcript spanning the CDS and $854 \mathrm{bp}$ of the $3^{\prime} \mathrm{UTR}$ (CDS+UTR). We observed that the presence of the $3^{\prime}$ UTR within the injected mRNA strongly decreases the impact of s1pr1 overexpression on vascular and cardiac defects (Figures 1, 2 compare CDS values with CDS+UTR values). These results indicate that s1pr1 might be negatively controlled at the post-transcriptional level at least during the first stages of zebrafish embryonic development.

In order to investigate whether s1pr1 might be controlled by miRNAs we used Targetscan to identify potential miRNA targets in the zebrafish s1pr1 $3^{\prime}$ UTR. Several targets were identified by this algorithm; however, our attention focused on miR-19a for several reasons: we recently highlighted a role for miR-19a in a zebrafish model of Holt-Oram (HOS), a pathology affecting 
heart and upper limbs (pectoral fins) both in mammals and fishes (Chiavacci et al., 2015). miR-19, as a component of the miR17-92 cluster, has been shown to be also involved in postnatal angiogenesis (Suarez et al., 2008) and to exert a pro angiogenic role in endothelial cell culture (Chamorro-Jorganes et al., 2016). Moreover, miR-19a,b are the miRNAs that obtain the highest score when matched to mouse and human s1prl UTR target sequences, according to Targetscan (www.targetscan.org/vert).

In order to verify whether miR-19a is able to modulate s1pr1 expression, the zebrafish s1pr1 3'UTR (GenBank: BC075741.1) was cloned downstream of the luciferase firefly reporter construct pGLU (Poliseno et al., 2010) creating the pGLU-s1pr1-3'UTR construct. HEK-293 cells were co-transfected with the pGLUs1pr1-3'UTR vector plus increasing doses of miR-19a mimic (Mi19a). As expected, the presence of the s1pr1 3'UTR sequence downstream of the luciferase coding sequence significantly decreased the reporter activity (Figure 4B). Additional increases in the amount of miR-19a produced further reductions in the relative luciferase activity in cells transfected with pGLUs1pr1-3'UTR vector but not in cells transfected with the control pGLU-s1pr1-3'UTRmut, in which 3 bases within the seed match sequence of miR-19a binding site were mutated (Figures 4A,C,D). Co-transfection of Mi-19a and miR-17-92 cluster sponge (a mRNA containing 2 repeats of the full miR17-92 cluster sequence) abolished the repressive effect exerted by this microRNA on the activity of the pGLU-s1pr1-3'UTR (Figure 4E). In line with these data, over-expression of miR-19a in zebrafish embryos significantly reduced the translational rate of a reporter construct (sensor), carrying GFP coding sequence upstream of $s 1$ pr1-3'UTR (Figure 4F). This reduction is almost erased if the mutated s1pr1-3'UTR is following the GFP sequence (Figure 4G).

These results indicate that 1 prl functions as a direct target of miR-19a.

\section{s1pr1 and miR-19a Functionally Interact in the Cardiac Context}

To further investigate the functional interaction between s1pr1 and miR-19a in the cardiac context, we assessed whether the morphological alterations generated by $\operatorname{spr} 1$ overexpression might be attenuated or exasperated by miR-19a increases or decreases, respectively. To modulate miR-19a levels, 1-cell stage $\operatorname{Tg}(m y l 7: E G F P)$ embryos were injected with Mi-19a or a specific morpholino against miR-19a (MO-19a) whose efficacy against miR-19a has been already shown (Chiavacci et al., 2015).

As we previously showed (Chiavacci et al., 2015), the increase of miR-19a alone negatively impacts cardiac development but not fin morphology (Figure 5A). However injection of 0.5 ng of Mi-19a together with 100 pg of full s1pr1 transcript significantly reduced the cardiac and fin defects caused by s1pr 1 overexpression. Conversely, the presence of Mi-19a exacerbates the cardiac and fin defects generated by the overexpression of s1pr1 CDS lacking the relevant 3 'UTR. Interestingly, co-injection of $\mathrm{Mi}-19 \mathrm{a}$ and s1pr1 CDS resulted in a synergistic negative interaction: while injection of either $0.5 \mathrm{ng}$ of $\mathrm{Mi}-19 \mathrm{a}$ or $50 \mathrm{pg}$ of s1pr1 CDS resulted respectively in 86 and $71 \%$ of $w t$-like hearts, the co-injection of Mi-19a and s1pr1 CDS at the same concentrations produced only the $45 \%$ of embryos displaying a $w t$-like phenotype and strongly increased the percentage of embryos with both mild and severe cardiac defects (Figure 5A). Although a specific analysis of cardiac genes controlled by miR$19 \mathrm{a}$ is not described, it is interesting to note that miR-19a has been reported to downregulate mef $2 \mathrm{ca}$ and mef2aa (Chiavacci et al., 2015). Therefore since our data indicate a negative impact of 1 pr 1 on mef2aa (Figure 3A), the synergistic repression of both miR-19a and s1pr1 on this gene which is highly expressed in zebrafish heart and involved in zebrafish heart function (Wang et al., 2005), might be one of the cause of the strong increase of cardiac defects observed in embryos co-injected with Mi-19a and s1pr1 CDS.

Although high doses (10 ng) of MO-19a did not affect embryo morphology when injected alone (Chiavacci et al., 2015) and (Figure 5B), it increased the severity of cardiac defects caused by s1pr1 overexpression. This effect was no longer detectable when only the CDS of s1pr1 was co-injected with MO-19a (Figure 5B). The decrease of miR-19a level had no significant effects on the fin morphology (Figure 5B).

Overall these data support a role of miR-19a as regulator of s1pr1 at least in the cardiac context.

\section{Downregulation of s1pr1 Partially Rescues Cardiac and Fin Defects Induced by Tbx5 Depletion in Zebrafish HOS Model}

We have recently shown that Tbx 5 depleted zebrafish embryos show a decreased level of miR-19a, and we demonstrated that miR-19a replacement partially rescues fin and cardiac defects caused by Tbx 5 depletion (Chiavacci et al., 2015). Therefore we hypothesized that the miR-19a downregulation might cause an increase of s1prl in heartstring (hst) embryos and that this increase might contribute to the HOS zebrafish phenotype. This hypothesis is supported by the observation that the phenotype of S1up embryos, including the altered heart morphology and the defective pectoral fins, was similar to the zebrafish mutant heartstrings (hst mut) (Garrity et al., 2002). To validate this hypothesis we first analyzed the s1pr1 expression by checking the recently generated list of genes differentially modulated in $w t$ - and Tbx5-depleted zebrafish embryos at 24 and $48 \mathrm{hpf}$ (Table S1, D’Aurizio et al., 2016). The microarray data do not indicate significant variations of s1pr1 expression in Tbx5depleted compared with $w t$ embryos at both developmental stages. However, in situ hybridization analysis performed in 48hpf embryos (Figure 6A) besides confirming the presence of slprl in fin buds and heart, shows a dominant s1prl expression in nervous tissues. This high nervous expression might prevent a reliable detection of relatively low cardiac modulations in a whole embryo analysis. Therefore we decided to quantify the s1pr1 expression in isolated hearts. This approach was performed by exploiting the $T b \times 5 a^{2296}$ mutant line which demonstrates a $G$ to $A$ transition at base pair 527. This change creates a nonsense mutation at the amino acid 120 within the Tbox region and is predicted to encoded a truncated protein, termed Tbx5a ${ }^{\mathrm{s} 296}$ (I. Scott and L. Parrie, unpublished data). 

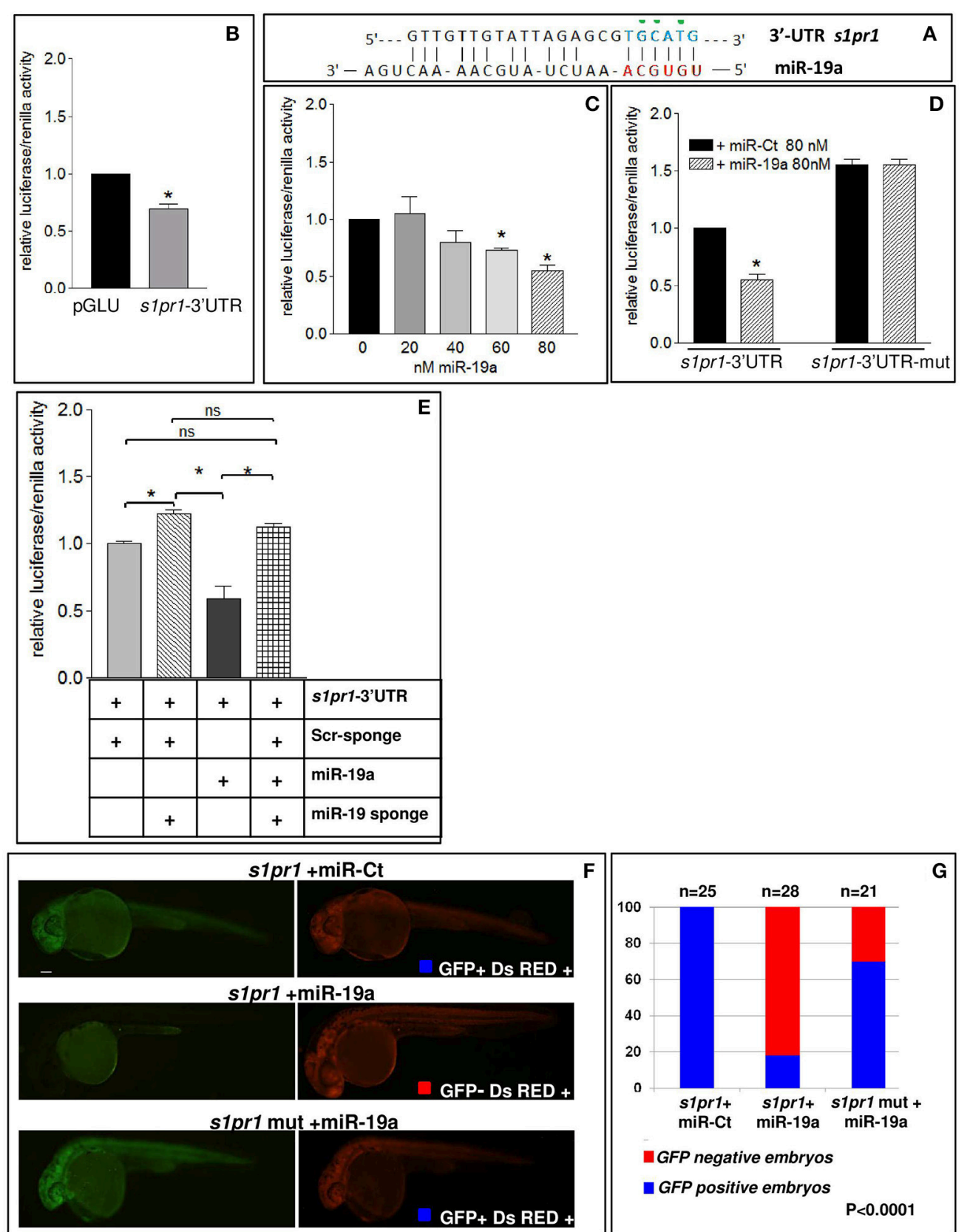

FIGURE 4 | s1pr1 is a direct target of miR-19a. (A) The computational alignment of a potential target site of miR-19a in s1pr1 of Danio rerio. The green dots indicate the bases mutated to destroy the seed sequence binding in the s1pr1-3' UTRmut construct. (B-E) The 3'UTR of dre-s1pr1 was cloned in the pGLU Dual-luciferase reporter plasmid (s1pr1-3'UTR) and transiently transfected into HK293 cells together with the Renilla luciferase pRL-TK vector as internal standard. The luciferase activity of s1pr1-3'UTR was compared: (B) with the luciferase activity of the empty vector; (C) in the presence of increasing amounts of miR-19a mimic; (D) with the luciferase activity of s1pr1-3'UTRmut (containing the s1pr1 3'UTR mutated in the seed match for miR-19a) in the presence of 80 nM of miR-19a mimic; (E) in the presence of $80 \mathrm{nM}$ of miR-19a mimic and $500 \mathrm{pg}$ of a sponge for miR-19a or $500 \mathrm{pg}$ of a scrambled sponge. In each transfection the total amount of the transfected miRNA was kept constant by adding a scrambled miRNA (miR-Ct) to the specific miRNA to obtain 80 ng. ${ }^{*} p<0.05$. (F,G) miR-19a negatively regulates the sensor s1pr1-GFP containing the wt3'UTR of s1pr1 downstream the GFP sequence but it is inefficient on the mut s1pr1-GFP; (F) representative 24 hpf embryos injected with wtor mut sensor mRNAs, miR-19a or miR-Ct mimics and DsRED mRNA. Left, miR-19a presence reduces the embryo specific fluorescence in the presence of wt3'UTR of s1pr1 and only the yolk autofluorescence is visible; right, DsRED expression is constant in all the experiment (G) quantification of the in vivo sensor assay. About 20 embryos were analyzed for each experiment. 


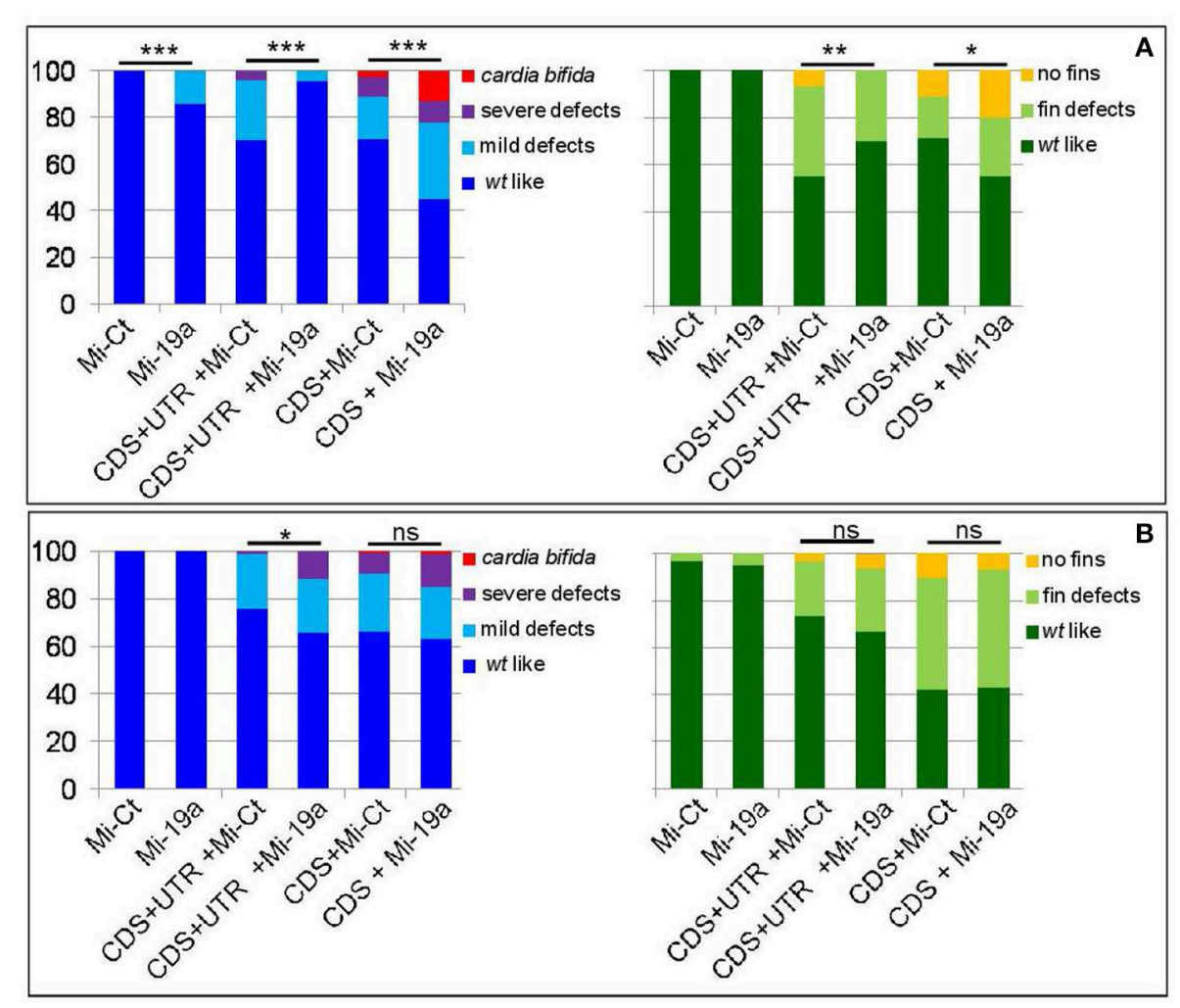

FIGURE 5 | miR-19a modulation impacts the fin and cardiac defects induced by the overexpression of s1pr1. Analysis at 72 hpf of Tg(Myl7:EGFP) embryos injected with CDS (50 pg) or CDS+UTR (100 pg) mRNA of s1pr1 in the presence of $0.5 \mathrm{ng}$ of miR-19a (Mi-19) or miR-Ct (Mi-Ct) mimics (A) or in the presence of $8 \mathrm{ng}$ of MO-19a or MO-Ct (B). For comparison the phenotypes of embryos injected only with Mi-19a or Mi-Ct or with MO-19a or MO-Ct are presented. The percentage of embryos with the indicated heart (left) or pectoral fin (right) defects was averaged across multiple independent experiments carried out in double blind. The total number of analyzed embryos were as follows: Mi-Ct injected = 120; Mi-19a injected = 99; s1pr1 CDS+UTR mRNA +Mi-Ct injected $n=133$; s1pr1 CDS+UTRmRNA + Mi-19a injected $n=142 ;$ s1pr1 CDS mRNA + Mi-Ct injected $n=155$; s1pr1 CDS mRNA + Mi-19a injected $n=148$; MO-Ct injected = 103; MO-19a injected $n=87$; s1pr1 CDS+UTR mRNA + MO-Ct injected $n=134$; s1pr1 CDS+UTR mRNA + MO-19a injected $n=156 ;$ s1pr1 CDS mRNA + MO-Ct injected $n=72$; s1pr1 CDS mRNA +MO-19a injected $n=65 .{ }^{\star} p<0.05,{ }^{\star \star} P<0.001,{ }^{\star \star \star *} P<0.0001$. For the other statistical analysis: (A) left, comparisons vs. Mi-Ct (line 3 and 5 vs. 1) have both $P<0.0001$, while comparisons vs. Mi- 19 has $P<0.05$ (line 4 vs. 2) and $P<0.0001$ (line 6 vs. 2); (B) left, all the comparisons (line 3 and 5 vs. 1 and line 4 and 6 vs. 2) have $P<0.0001$; (A,B) right, all the comparisons (line 3 and 5 vs. 1 and line 4 and 6 vs. 2) have $P<0.0001$.

Embryos homozygous for Tbx $5 \mathrm{a}^{\text {s2 } 296}$ develop the hst phenotype and lack any evidence of pectoral fins. Hearts were dissected from 72 hpf embryos obtained from crossing Tbx5a ${ }^{\text {s296/+ }}$ parent line. We dissected hearts with the hst phenotype (from Tbx5a s296/s296 embryos which lack pectoral fins) or with $w t$ phenotype (from Tbx5a s296/+ or Tbx5a+/+ siblings showing normal fins) as controls. Total RNA was extracted from hst and normal sibling hearts and the level of s1pr1 transcript was analyzed by digital droplet PCR (ddPCR; Data Sheet 2). This analysis, which allowed us to accurately quantify a specific transcript, shows a 3-fold increase of s1prl cardiac expression in hst compared to normal hearts (Figure 6B). Next, to verify if this increase could contribute to the HOS phenotype we performed rescue experiments. Figures 6C,D shows that co-injection of $1.5 \mathrm{ng}$ of MO-Tbx5a with $0.2 \mathrm{ng}$ of the MO-s1pr1 but not with the same quantity of the control morpholino, resulted in significantly fewer cardiac and fin defects. Injection of $0.2 \mathrm{ng}$ of MO-s1pr1 alone does not affect embryo morphology to the degree that the $0.5 \mathrm{ng}$ dose does (not shown); however higher dosage of MO-s1pr1 worsens the morphological alterations of HOS embryos (Figure 6C) suggesting that defined dosage of s1pr1 is essential for the correct development of the heart.

To further support a role of 1 pr 1 in Tbx 5 regulatory circuit we treated Tbx5a morphants or non-injected embryos with the specific s1pr1 inhibitor (R)-3-amino-4-(3-hexylphenylamino)4-oxobutylphosphonic acid trifluoroacetate (W146) (Tarrason et al., 2011; Kunkel et al., 2013) or only with its vehicle. We used a large range of doses for this approach in light of the fact that there are no data concerning the use of W146 in zebrafish. However a progressive and dose dependent increase of vascular defects which we observed in zebrafish embryos was in accord with the s1pr1 antagonist role played by this drug (not shown). Injection of $2.5 \mathrm{ng} / \mathrm{ml}$ of MO-Tbx 5 generated heart defects in $\sim 90 \%$ of embryos. Morphant embryos exposed to 0.05 to $1 \mathrm{ug} / \mathrm{ml}$ of W146 demonstrated a statistically lower incidence of heart defects (Figure 6E). In contrast, the incidence of heart defects in non-injected control embryos exposed to W146 was low and did not differ from the no-drug controls. 


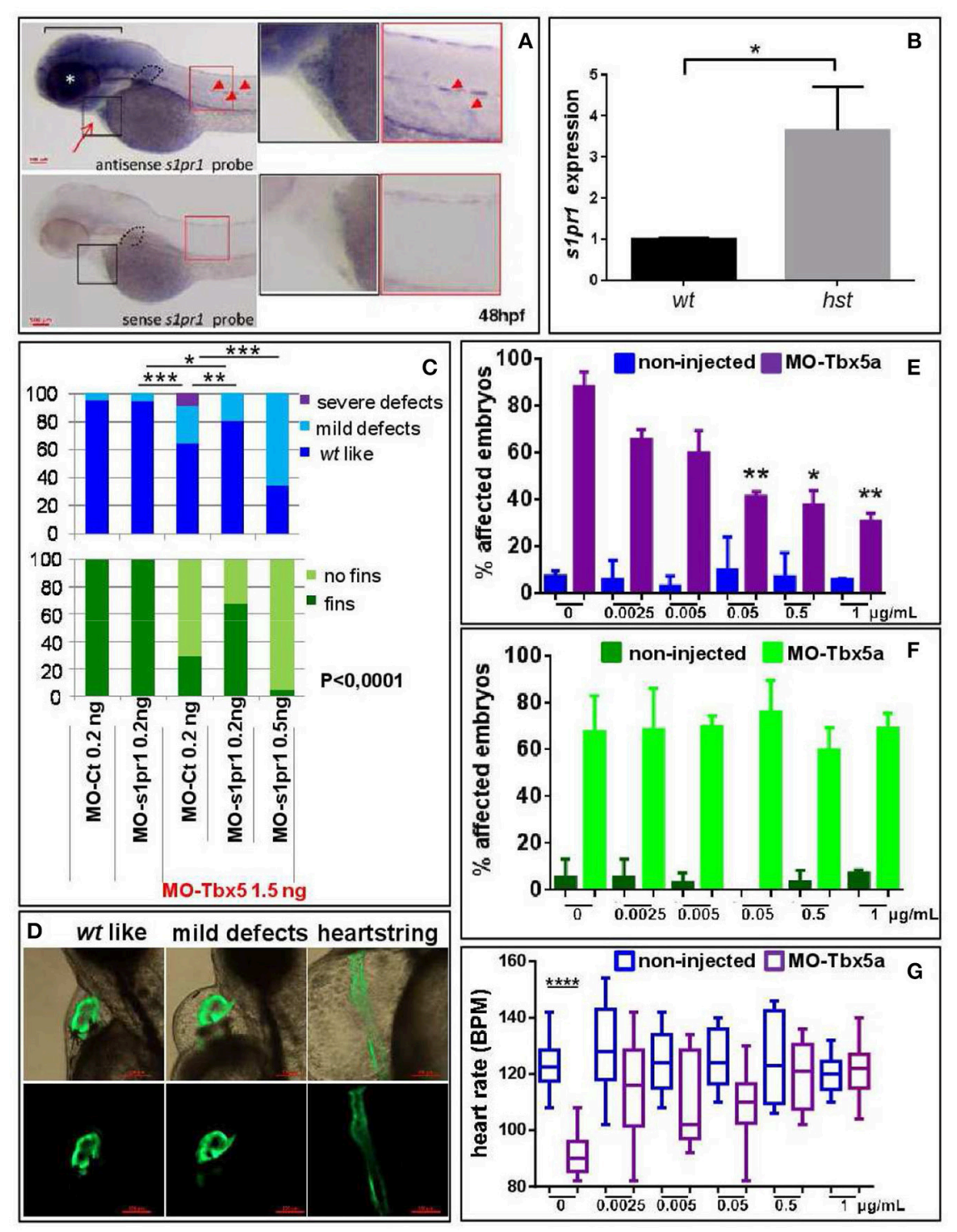

FIGURE 6 | s1pr1 is overexpressed in zebrafish HOS hearts and its downregulation is able to partially rescue heart/fin defects induced by Tbx5 depletion. (A) Examples of s1pr1 ISH performed on 48 hpf embryos. Top left, arrow and arrowheads indicate respectively the hybridization signals at the heart and vascular levels; dotted line encircles fin bud, bracket and asterisk mark respectively the hybridization signal on brain and eye areas Bottom left, control ISH performed with sense s1pr1 probe. Right, magnifications of the cardiac and dorsal areas. Scale bar $=100 \mu \mathrm{m}$. (B) Quantification by ddPCR of s1pr1 transcript in hearts dissected from 72 hpf normal and tbx $5 a^{296}$ mutant embryos. Data were normalized on ef $1 \alpha$ as internal standard and are relative to wt sets as 1 . Three different experiments starting from 3 different clutches were used for this analysis. (C,D) Rescue of Tbx5 morphants by s1pr1 depletion. (C) Analysis of $72 \mathrm{hpf}$ Tbx5 morphants. MO-s1pr1 or MO-Ct at the reported doses, were co-injected with $1.5 \mathrm{ng}$ of MO-Tbx5a in Tg(Myl7:EGFP)embryos. For comparison the phenotypes of embryos injected only with $\mathrm{MO}-\mathrm{s} 1 \mathrm{pr} 1$ or $\mathrm{MO}-\mathrm{Ct}$ are presented. The percentage of embryos with the indicated heart (top) or pectoral fin (bottom) defects was averaged across multiple independent experiments carried out in double blind. All the comparisons indicated in the heart graph were highly significant in fin graphs $(P<0.0001)$. The total number of analyzed Tbx5 morphant embryos were as follows: MO-Ct co-injected $n=136$ (0.2 ng), $n=137$ (0.5 ng); MO-s1pr1 co-injected $n=136$ (0.2 ng), $n=203$ (0.5 ng). One hundred and fifty embryos were injected only with MO-Ct and 50 only with MO-s1pr1. (D) Some examples of the different cardiac phenotypes obtained in the experiments presented in (C). ${ }^{\star} p<0.05,{ }^{\star \star} P<0.001,{ }^{\star \star \star} P<0.0001$. (E-G) Rescue of Tbx5 morphants by s1pr1 chemical inhibition. 2.5 ng of MO-Tbx5a was injected into Tg(Myl7:EGFP)embryos. At 24 hpf, W146 was added at the indicated doses to E3 medium of morphants and non-injected embryos. (E) Cardiac morphology analysis of 72 hpf Tbx5a morphants and non-injected embryos. Affected embryos either had heartstrings morphology, pericardial edema, or looping/chamber defects. The total number of analyzed Tbx5 morphant embryos were as follows: $0 \mu \mathrm{g} n=51,0.0025 \mu \mathrm{g} n=32,0.005 \mu \mathrm{g} n=27,0.05 \mu \mathrm{g} n=29$, $0.5 \mu \mathrm{g} n=34,1 \mu \mathrm{g} n=32$. The total number of analyzed non-injected control embryos were as follows: $0 \mu \mathrm{g} n=28,0.0025 \mu \mathrm{g} n=38,0.005 \mu \mathrm{g} n=30,0.05 \mu \mathrm{g}$ $n=31,0.5 \mu \mathrm{g} n=31,1 \mu \mathrm{g} n=34 .{ }^{*} P<0.05$, ${ }^{\star \star} P<0.001$ vs. morphants with $0 \mu \mathrm{g} / \mathrm{mL}$ W146. None the non-injected control embryos treated with W146 showed any significant differences compared to embryos receiving $0 \mu \mathrm{g} / \mathrm{mL}$ W146. (F) Fin analysis of 72 hpf Tbx5a morphants and non-injected embryos. Fins were scored for the presence of both pectoral fins. No statistical differences were observed among drug application groups for either non-injected and Tbx5 morphant embryos, compared to no drug controls. (G) Heart rate analysis of 72 hpf Tbx5a morphants and non-injected embryos. Ten embryos were scored for each group as described in section Materials and Methods. 
On the contrary none of the tested doses was able to cause fin rescue (Figure 6F). To further confirm the positive effect of W146 in the cardiac context we analyzed the heart rate. The Tbx5 morphants present a heart rate significantly lower compared to control embryos, which is consistent with Tbx 5 mutants (Garrity et al., 2002). While the lowest doses of W146 (0.0025-0.05 $\mathrm{ug} / \mathrm{ml}$ ) produced a variable increase in heart rate that did not reach statistical significance, the two higher doses of 0.5 or 1 $\mathrm{ug} / \mathrm{ml}$ consistently restored the heart rate of Tbx 5 morphants to statistically normal levels (Figure 6G). As expected, in the noninjected controls no heart rate differences were observed for any dose of W146.

Overall these data support the hypothesis that one of the negative consequences of Tbx $5 \mathrm{a}$ depletion in zebrafish is an increase of s1pr1 expression as consequence of miR-19a downregulation.

\section{DISCUSSION}

In this study we have uncovered a role of s1pr1 in zebrafish cardiac development. Data obtained from s1prl loss-of-function studies in zebrafish are rather contradictory (Ben Shoham et al., 2012; Gaengel et al., 2012; Tobia et al., 2012; Mendelson et al., 2013; Hisano et al., 2015). To investigate the role of this receptor in zebrafish we performed gain of function experiments overexpressing s1pr1 to overcome both the possible functional redundancy among the $\mathrm{s} 1 \mathrm{P}$ receptor family members and the concern about the use of morpholinos in this context.

Overexpression of s1prl causes defects in ISV morphology and CVP confirming the contribution of this receptor to vascular patterning, but also generates incorrect cardiac looping and alterations of cardiac chamber morphology demonstrating a previously unappreciated role of s1pr1 in heart development. At the higher dosages of injected s1pr1, the presence of a few cases of cardia bifida suggest its possible involvement in cardiac precursor migration through mechanisms which would be interesting to investigate. We observed a slight but significant upregulation of s1pr2 in hearts isolated from s1pr1 overexpressing hearts compared to control hearts. Although s1pr2 mutation/downregulation is known to prevents cardiac precursors migrating thus resulting in cardia bifida (Kupperman et al., 2000), there are no data about the consequences of s1pr2 upregulation. Therefore at the moment we can only hypothesize that s1pr2 upregulation might contribute to the cardiac defects observed in s1pr1 overexpressing embryos.

Disruption of the vascular network frequently causes circulatory defects and defective circulation can be cause of pericardiac edema and abnormal heart looping. Although we cannot exclude that in s1pr1 overexpressing embryos circulation defects could, at some level, influence cardiac morphology, some considerations support a direct role of s1pr1 in the cardiac context. First, we showed that overexpression of s1pr1 influences the myocardial precursor pool before heart tube formation (Figures 3B,C). Moreover, low s1pr1 dosages which do not affect vascular development and do not alter circulation, are nevertheless able to affect cardiac morphology (Figures 1, 2). Lastly, injection of the morpholino against s1pr1 (MO-s1pr1) which strongly affects blood flow (as also reported in several papers), has minimal effects on heart development.

Interestingly, our analysis also highlights that $s 1$ prl is subject to a negative post-transcriptional control by miR-19a. This effect has some important implications. The first one is that the activity of this receptor is hampered in the cardiac and vascular contexts where miR-19a is expressed, at least during the first stages of zebrafish development. In line with this observation a further decrease of s1pr1 levels by MO-s1pr1 injection has a very limited impact on cardiac development (Figure S1) although it is known to strongly alter vascular morphology (Ben Shoham et al., 2012; Gaengel et al., 2012; Tobia et al., 2012; Mendelson et al., 2013). The role of miR-19a in s1pr1 regulation has been functionally demonstrated: (1) by the observation that microinjections of the same quantities of s1pr1 mRNAs have significantly different impacts on cardiovascular development depending on whether the mRNA includes its 3 'UTR or not (Figure 2); (2) by showing that miR-19a gain or loss of function is able to respectively decrease or increase cardiovascular defects generated by s1pr1 over-expression, only when its full length mRNA sequence has been injected. These findings corroborate the importance and the pervasiveness of microRNA-mediated regulatory control in the cardiovascular context. They also highlight the importance of transfecting the full length mRNA sequence of a gene under study to obtain a faithful picture of its biological impact, rather than injecting only the CDS sequence as was described for prior gain of function experiments performed with s1pr1 (Mendelson et al., 2013).

The second important consequence of the posttranscriptional control of s1prl by miR-19a is that since this microRNA is regulated by Tbx5 in zebrafish embryos (Chiavacci et al., 2015) then s1pr1 is indirectly under the Tbx5 control and may contribute to the zebrafish HOS phenotype caused by Tbx5 depletion. The engagement of s1pr1 in the Tbx5 regulatory circuit is supported by data showing an increase of this receptor in zebrafish Tbx5a mutant embryos and by rescue experiments. Both s1pr1 depletion or inhibition by respectively morpholino or antagonist administration are able to decrease the level of cardiac defects. However while MO-s1pr1 injection positively impacts also fin morphology, this effect was not observed as a consequence of antagonist exposure. At the moment we have no explanation for this difference and we can only hypothesize that different tissue context might account for differences in drug susceptibility and/or toxicity.

Very recently, two new frame shift Tbx5 alleles have been generated using CRISPR-Cas9 mutagenesis. These mutants show the heart and fin defects which characterize hst mutants and morpholino knockdown, but do not present the strong heartstrings phenotype (Chiavacci et al., 2017). In light of these data which underlines the importance of analyzing several individual alleles of a candidate gene to evaluate its functions, it will be interesting to evaluate the impact 
of miR-19a and s1prl modulation in all these different classes of mutants.

Our results, such as many others concerning s1pr1, are in contrast with data showing the absence of a clear phenotype in zebrafish mutants for this receptor (Hisano et al., 2015). However differences between the phenotypes caused by genetic mutations and those caused by gene knockdowns have been reported mainly in zebrafish where the use of reverse genetics is dramatically increased (Kok et al., 2015), but also in other model systems. While at the beginning these differences were interpreted as off target consequences of morpholino activity, more recently several experimental data point to the activation of compensatory networks. These networks are able to counterbalance deleterious mutations but appear not to be induced in response to translational or transcriptional knockdown (Rossi et al., 2015). The molecular mechanisms responsible of the different responses of mutants vs. morphants is object of debate, although a nonsense-mediated mRNA decay (NMD) activated by sequence alteration is suggested as a possible mechanism of induction (Rossi et al., 2015). In the case of $s 1$ pr 1 , the existence of several s1pr members with partial functional redundancy might offer an easy context for molecular compensation.

\section{ETHICS STATEMENT}

The corresponding author declares that all experiments were performed in accordance with relevant guidelines and regulations. The zebrafish facility, where all the experiments have been performed, is part of the CENTRO di BIOMEDICINA SPERIMENTALE (CBS) of the Area della Ricerca del CNR, Via Moruzzi 1, 56124 Pisa. The zebrafish facility has been authorized by the Italian Ministry of Health with the authorization $\mathrm{n}^{\circ} 297 / 2012$-A of the $12 / 21 / 2012$. The corresponding author declares that all the methods were carried out in accordance with the approved guidelines and that all experimental protocols were approved by the Italian Ministry of Health. Moreover all

\section{REFERENCES}

Agostini, S., Chiavacci, E., Matteucci, M., Torelli, M., Pitto, L., and Lionetti, V. (2015). Barley beta-glucan promotes MnSOD expression and enhances angiogenesis under oxidative microenvironment. J. Cell. Mol. Med. 19, 227-238. doi: $10.1111 /$ jcmm. 12442

Alewijnse, A. E., Peters, S. L., and Michel, M. C. (2004). Cardiovascular effects of sphingosine-1-phosphate and other sphingomyelin metabolites. Br. J. Pharmacol. 143, 666-684. doi: 10.1038/sj.bjp.0705934

Allende, M. L., and Proia, R. L. (2002). Sphingosine-1-phosphate receptors and the development of the vascular system. Biochim. Biophys. Acta 1582, 222-227. doi: 10.1016/S1388-1981(02)00175-0

Auman, H. J., Coleman, H., Riley, H. E., Olale, F., Tsai, H. J., and Yelon, D. (2007). Functional modulation of cardiac form through regionally confined cell shape changes. PLoS Biol. 5:e53. doi: 10.1371/journal. pbio.0050053

Bartel, D. P. (2009). MicroRNAs: target recognition and regulatory functions. Cell 136, 215-233. doi: 10.1016/j.cell.2009.01.002

Begemann, G., and Ingham, P. W. (2000). Developmental regulation of Tbx5 in zebrafish embryogenesis. Mech. Dev. 90, 299-304. doi: 10.1016/S0925-4773(99)00246-4 the technicians and researcher who take care of the animals and perform the experiments are appropriately trained by attending specific courses.

\section{AUTHOR CONTRIBUTIONS}

LP designed the experiments; EG performed the most part of the microinjection experiments in zebrafish and the ISH; EC performed a minor part of the microinjection experiments in zebrafish; NA performed heart dissectioning of hst mutants and the ddPCR analysis; CI performed confocal imaging; MR performed QRT analysis; ME and LM realized the experiments on cell culture; LP wrote the manuscript; DG and FC revised the manuscript. MR and FC financed the work. All authors read and approved the final version of the paper.

\section{FUNDING}

The work was partially supported by Istituto Toscano Tumori (grant 2013-MR). The work was partially supported by University and Research grant PRIN-2102 (FC) Scuola Superiore di Studi SAnna supported the salary of EG as Ph.D. student and contributed to some research costs.

\section{ACKNOWLEDGMENTS}

We thank Roberto Ripa (Scuola Normale Superiore di Pisa) for kindly providing the miR-17-92 cluster sponge.

\section{SUPPLEMENTARY MATERIAL}

The Supplementary Material for this article can be found online at: https://www.frontiersin.org/articles/10.3389/fcell.2018. 00058/full\#supplementary-material

Data Sheet 1 | Original Q-RT-PCR data relative to the analysis presented in Figure 3A.

Data Sheet 2 | Original ddPCR data relative to the analysis presented in Figure 6B.

Beis, D., Bartman, T., Jin, S. W., Scott, I. C., D’Amico, L. A., Ober, E. A., et al. (2005). Genetic and cellular analyses of zebrafish atrioventricular cushion and valve development. Development 132, 4193-4204. doi: 10.1242/dev.01970

Ben Shoham, A., Malkinson, G., Krief, S., Shwartz, Y., Ely, Y., Ferrara, N., et al. (2012). S1P1 inhibits sprouting angiogenesis during vascular development. Development 139, 3859-3869. doi: 10.1242/dev.078550

Blaho, V. A., and Hla, T. (2011). Regulation of mammalian physiology, development, and disease by the sphingosine 1-phosphate and lysophosphatidic acid receptors. Chem. Rev. 111, 6299-6320. doi: 10.1021/cr200273u

Chae, S. S., Paik, J. H., Allende, M. L., Proia, R. L., and Hla, T. (2004). Regulation of limb development by the sphingosine 1-phosphate receptor S1p1/EDG-1 occurs via the hypoxia/VEGF axis. Dev. Biol. 268, 441-447. doi: 10.1016/j.ydbio.2004.01.001

Chamorro-Jorganes, A., Lee, M. Y., Araldi, E., Landskroner-Eiger, S., Fernandez-Fuertes, M., Sahraei, M., et al. (2016). VEGF-Induced Expression of miR-17-92 Cluster in Endothelial Cells Is Mediated by ERK/ELK1 Activation and Regulates Angiogenesis. Circ. Res. 118, 38-47. doi: 10.1161/CIRCRESAHA.115.307408

Chiavacci, E., D’Aurizio, R., Guzzolino, E., Russo, F., Baumgart, M., Groth, M., et al. (2015). MicroRNA 19a replacement partially rescues fin and 
cardiac defects in zebrafish model of Holt Oram syndrome. Sci. Rep. 5:18240. doi: $10.1038 /$ srep 18240

Chiavacci, E., Dolfi, L., Verduci, L., Meghini, F., Gestri, G., Evangelista, A. M. M., et al. (2012). MicroRNA 218 mediates the effects of Tbx5a over-expression on Zebrafish heart development. PLOS ONE 7:e50536. doi: 10.1371/journal.pone.0050536

Chiavacci, E., Kirchgeorg, L., Felker, A., Burger, A., and Mosimann, C. (2017). Early frameshift alleles of zebrafish tbx5a that fail to develop the heartstrings phenotype. bioRxiv. doi: 10.1101/103168

Chun, J., Goetzl, E. J., Hla, T., Igarashi, Y., Lynch, K. R., Moolenaar, W., et al. (2002). International Union of Pharmacology. XXXIV. Lysophospholipid receptor nomenclature. Pharmacol. Rev. 54, 265-269. doi: 10.1124/pr.54.2.265

Clay, H., Wilsbacher, L. D., Wilson, S. J., Duong, D. N., McDonald, M., Lam, I., et al. (2016). Sphingosine 1-phosphate receptor-1 in cardiomyocytes is required for normal cardiac development. Dev. Biol. 418, 157-165. doi: 10.1016/j.ydbio.2016.06.024

Cordes, K. R., and Srivastava, D. (2009). MicroRNA regulation of cardiovascular development. Circ. Res. 104, 724-732. doi: 10.1161/CIRCRESAHA.108.192872

D'Aurizio, R., Russo, F., Chiavacci, E., Baumgart, M., Groth, M., D'Onofrio, M., et al. (2016). Discovering miRNA regulatory networks in HoltOram syndrome using a Zebrafish model. Front. Bioeng. Biotechnol. 4:60. doi: 10.3389/fbioe.2016.00060

Dietrich, A. C., Lombardo, V. A., Veerkamp, J., Priller, F., and AbdelilahSeyfried, S. (2014). Blood flow and Bmp signaling control endocardial chamber morphogenesis. Dev. Cell 30, 367-377. doi: 10.1016/j.devcel.2014.06.020

Gaengel, K., Niaudet, C., Hagikura, K., Lavina, B., Muhl, L., Hofmann, J. J., et al. (2012). The sphingosine-1-phosphate receptor S1PR1 restricts sprouting angiogenesis by regulating the interplay between VE-cadherin and VEGFR2. Dev. Cell 23, 587-599. doi: 10.1016/j.devcel.2012.08.005

Garrity, D. M., Childs, S., and Fishman, M. C. (2002). The heartstrings mutation in zebrafish causes heart/fin Tbx5 deficiency syndrome. Development 129, $4635-4645$.

Ghosh, T. K., Song, F. F., Packham, E. A., Buxton, S., Robinson, T. E., Ronksley, J., et al. (2009). Physical interaction between TBX5 and MEF2C is required for early heart development. Mol. Cell. Biol. 29, 2205-2218. doi: 10.1128/MCB.01923-08

Hisano, Y., Inoue, A., Taimatsu, K., Ota, S., Ohga, R., Kotani, H., et al. (2015). Comprehensive analysis of sphingosine-1-phosphate receptor mutants during zebrafish embryogenesis. Genes Cells 20, 647-658. doi: 10.1111/gtc.12259

Huang, C. J., Tu, C. T., Hsiao, C. D., Hsieh, F. J., and Tsai, H. J. (2003). Germline transmission of a myocardium-specific GFP transgene reveals critical regulatory elements in the cardiac myosin light chain 2 promoter of zebrafish. Dev. Dyn. 228, 30-40. doi: 10.1002/dvdy. 10356

Kimmel, C. B., Ballard, W. W., Kimmel, S. R., Ullmann, B., and Schilling, T. F. (1995). Stages of embryonic development of the zebrafish. Dev. Dyn. 203, 253-310. doi: 10.1002/aja.1002030302

Kok, F. O., Shin, M., Ni, C. W., Gupta, A., Grosse, A. S., van Impel, A., et al. (2015). Reverse genetic screening reveals poor correlation between morpholino-induced and mutant phenotypes in zebrafish. Dev. Cell 32, 97-108. doi: 10.1016/j.devcel.2014.11.018

Kunkel, G. T., Maceyka, M., Milstien, S., and Spiegel, S. (2013). Targeting the sphingosine-1-phosphate axis in cancer, inflammation and beyond. Nat. Rev. Drug Discov. 12, 688-702. doi: 10.1038/nrd4099

Kupperman, E., An, S., Osborne, N., Waldron, S., and Stainier, D. Y. (2000). A sphingosine-1-phosphate receptor regulates cell migration during vertebrate heart development. Nature 406, 192-195. doi: 10.1038/35018092

Levade, T., Auge, N., Veldman, R. J., Cuvillier, O., Negre-Salvayre, A., and Salvayre, R. (2001). Sphingolipid mediators in cardiovascular cell biology and pathology. Circ. Res. 89, 957-968. doi: 10.1161/hh2301.100350

Liu, Y., Wada, R., Yamashita, T., Mi, Y., Deng, C. X., Hobson, J. P., et al. (2000). Edg-1, the G protein-coupled receptor for sphingosine-1-phosphate, is essential for vascular maturation. J. Clin. Invest. 106, 951-961. doi: 10.1172/JCI10905

Lu, F., Langenbacher, A. D., and Chen, J. N. (2016). Transcriptional regulation of heart development in zebrafish. J. Cardiovasc. Dev. Dis. 3:14. doi: $10.3390 /$ jcdd 3020014

Means, C. K., and Brown, J. H. (2009). Sphingosine-1-phosphate receptor signalling in the heart. Cardiovasc. Res. 82, 193-200. doi: 10.1093/cvr/cvp086

Mendelson, K., Zygmunt, T., Torres-Vazquez, J., Evans, T., and Hla, T. (2013). Sphingosine 1-phosphate receptor signaling regulates proper embryonic vascular patterning. J. Biol. Chem. 288, 2143-2156 doi: 10.1074/jbc.M112.427344

Mizugishi, K., Yamashita, T., Olivera, A., Miller, G. F., Spiegel, S., and Proia, R. L. (2005). Essential role for sphingosine kinases in neural and vascular development. Mol. Cell. Biol. 25, 11113-11121. doi: 10.1128/MCB.25.24.11113-11121.2005

Parrie, L. E., Renfrew, E. M., Wal, A. V., Mueller, R. L., and Garrity, D. M. (2013). Zebrafish tbx5 paralogs demonstrate independent essential requirements in cardiac and pectoral fin development. Dev. Dyn. 242, 485-502. doi: $10.1002 /$ dvdy.23953

Poliseno, L., Salmena, L., Riccardi, L., Fornari, A., Song, M. S., Hobbs, R. M., et al. (2010). Identification of the miR-106b 25 microRNA cluster as a proto-oncogenic PTEN-targeting intron that cooperates with its host gene MCM7 in transformation. Sci Signal 3:ra29. doi: 10.1126/scisignal.20 00594

Reiter, J. F., Alexander, J., Rodaway, A., Yelon, D., Patient, R., Holder, N., et al. (1999). Gata5 is required for the development of the heart and endoderm in zebrafish. Genes Dev. 13, 2983-2995. doi: 10.1101/gad.13.22.2983

Rossi, A., Kontarakis, Z., Gerri, C., Nolte, H., Holper, S., Kruger, M., et al. (2015). Genetic compensation induced by deleterious mutations but not gene knockdowns. Nature 524, 230-233. doi: 10.1038/nature14580

Ruvinsky, I., Oates, A. C., Silver, L. M., and Ho, R. K. (2000). The evolution of paired appendages in vertebrates: T-box genes in the zebrafish. Dev. Genes Evol. 210, 82-91. doi: 10.1007/s004270050014

Serbedzija, G. N., Chen, J. N., and Fishman, M. C. (1998). Regulation in the heart field of zebrafish. Development 125, 1095-1101.

Singleman, C., and Holtzman, N. G. (2011). Heart dissection in larval, juvenile and adult zebrafish, Danio rerio. J. Vis. Exp. doi: 10.3791/3165. [Epub ahead of print].

Suarez, Y., Fernandez-Hernando, C., Yu, J., Gerber, S. A., Harrison, K. D., Pober, J. S., et al. (2008). Dicer-dependent endothelial microRNAs are necessary for postnatal angiogenesis. Proc. Natl. Acad. Sci. U.S.A. 105, 14082-14087. doi: $10.1073 /$ pnas. 0804597105

Tarrason, G., Auli, M., Mustafa, S., Dolgachev, V., Domenech, M. T., Prats, N., et al. (2011). The sphingosine-1-phosphate receptor-1 antagonist, W146, causes early and short-lasting peripheral blood lymphopenia in mice. Int. Immunopharmacol. 11, 1773-1779. doi: 10.1016/j.intimp.2011. 07.004

Tobia, C., Chiodelli, P., Nicoli, S., Dell'era, P., Buraschi, S., Mitola, S., et al. (2012). Sphingosine-1-phosphate receptor-1 controls venous endothelial barrier integrity in zebrafish. Arterioscler. Thromb. Vasc. Biol. 32, e104-e116. doi: 10.1161/ATVBAHA.112.250035

van Rooij, E., Marshall, W. S., and Olson, E. N. (2008). Toward microRNA-based therapeutics for heart disease: the sense in antisense. Circ. Res. 103, 919-928. doi: 10.1161/CIRCRESAHA.108.183426

Verduci, L., Simili, M., Rizzo, M., Mercatanti, A., Evangelista, M., Mariani, L., et al. (2010). MicroRNA (miRNA)-mediated interaction between leukemia/lymphoma-related factor (LRF) and alternative splicing factor/splicing factor 2 (ASF/SF2) affects mouse embryonic fibroblast senescence and apoptosis. J. Biol. Chem. 285, 39551-39563. doi: 10.1074/jbc.M110.114736

Wang, Y. X., Qian, L. X., Yu, Z., Jiang, Q., Dong, Y. X., Liu, X. F., et al. (2005). Requirements of myocyte-specific enhancer factor $2 \mathrm{~A}$ in zebrafish cardiac contractility. FEBS Lett. 579, 4843-4850. doi: 10.1016/j.febslet.2005.07.068

Yelon, D., Ticho, B., Halpern, M. E., Ruvinsky, I., Ho, R. K., Silver, L. M., et al. (2000). The bHLH transcription factor hand2 plays parallel roles in zebrafish heart and pectoral fin development. Development 127, 2573-2582.

Conflict of Interest Statement: The authors declare that the research was conducted in the absence of any commercial or financial relationships that could be construed as a potential conflict of interest.

Copyright (c) 2018 Guzzolino, Chiavacci, Ahuja, Mariani, Evangelista, Ippolito, Rizzo, Garrity, Cremisi and Pitto. This is an open-access article distributed under the terms of the Creative Commons Attribution License (CC BY). The use, distribution or reproduction in other forums is permitted, provided the original author(s) and the copyright owner are credited and that the original publication in this journal is cited, in accordance with accepted academic practice. No use, distribution or reproduction is permitted which does not comply with these terms. 